\title{
Occult Fractures in the Carpal Region: Incidental Findings on Bone Scintigraphy
}

\author{
Sahel Zoakman ${ }^{1}$, Roderick van Leerdam ${ }^{2}$, Frank Beeres ${ }^{3}$, Steven Rhemrev ${ }^{3}$ \\ ${ }^{1}$ Emergency Department, Het Röpke-Zweers Ziekenhuis, Hardenberg, The Netherlands; ${ }^{2}$ Department of Surgery, Groene Hart \\ Ziekenhuis, Gouda, The Netherlands; ${ }^{3}$ Department of Surgery, Medisch Centrum Haaglanden, The Hague, The Netherlands. \\ Email: sahel_zoakman@live.nl
}

Received January $9^{\text {th }}$, 2013; revised February $10^{\text {th }}$, 2013; accepted February $20^{\text {th }}, 2013$

\begin{abstract}
The aim of this study was to evaluate the number and distribution of fractures around the wrist found on bone scintigraphy in patients with a clinically suspected scaphoid fracture and negative initial radiographs. We retrospectively included 445 consecutive patients with a suspected scaphoid fracture who underwent routine bone scintigraphy. None of the radiographs showed evidence of a fracture. We analyzed the type and number of other fractures incidentally found on bone scintigraphy. On average, bone scintigraphy was done in 4 days $(1-9)$. The outcome of bone scintigraphy: 80 (18.0\%) a scaphoid fracture, 145 (32.6\%) another fracture in the carpal region, 208 (46.7\%) normal and the diagnosis of 12 (2.8\%) was unclear. In the present study, we demonstrated that in patients with a clinically suspected scaphoid fracture and negative initial radiographs, bone scintigraphy detected in many cases (64.4\%) other fractures in the carpal region. This suggests that radiographs not only miss scaphoid but also many other carpal and distal radius fractures. Solutions should be found to solve this problem and probably advanced imaging techniques like CT, MRI or bone scintigraphy should be used in the correct clinical scenario.
\end{abstract}

Keywords: Wrist Injury; Carpal Fractures; Bone Scintigraphy; Scaphoid Fracture; Scaphoid Radiographs

\section{Introduction}

Fractures in and around the wrist are common [1]. The evaluation of patients with wrist injuries is a diagnostic challenge. In many cases the clinical examination does not provide diagnostic certainty and radiographs traditionally used in the initial investigation might be negative as some fractures are often occult on X-rays $[2,3]$. Injuries of the wrist lead to high morbidity and disability. Early detection and treatment for example of a scaphoid fracture is important to prevent long term complications such as non-union, osteonecrosis, carpal instability and osteoarthropathy. If a fracture is suspected, there is a need for a fast and reliable diagnostic method. Bone scintigraphy has been used in diagnosing fractures at all sites since the 1960s [4].

Patients who visited the Emergency Department with an acute trauma and a suspected scaphoid fracture after physical examination but no evidence of a scaphoid fracture on plain radiographs underwent at our institution bone scintigraphy [5-11]. We noticed that bone scintigraphy detected many other occult carpal and distal radius fractures in these patients with a suspected scaphoid fracture. It is therefore interesting to evaluate how often these other fractures are not caught on conventional radiographs. Consequently the aim of this study was to evaluate the number and distribution of fractures around the wrist found on bone scintigraphy in patients with a clinically suspected scaphoid fracture and negative initial radiographs.

\section{Patients and Methods}

In this study the prospectively obtained data of 445 patients with suspected scaphoid fractures between March 2004 and April 2011 were included and analyzed. All the patients gave the informed consent prior being included into the study. The study was authorized by the local ethical committee and was performed in accordance with the Ethical standards of the 1964 Declaration of Helsinki as revised in 2000. All data was collected by reviewing the standardised reports. Patients were eligible if they consented to participate, had a suspected scaphoid fracture after a recent trauma (within 48 hours) and no evidence of a fracture on plain radiographs. Patients who had contraindications for bone scintigraphy were excluded. The SPSS Statistics software (Version 17.0) was used for all statistical calculations.

\section{Results}

Between March 2004 and April 2011, 445 consecutive 
patients attended the emergency department with a history and signs consistent with a fractured scaphoid. There were 207 male and 238 female patients, the age range was 10 - 88 years (mean 39 years). All patients received conventional radiography at presentation in the Emergency Department which showed no fracture. Radiography was not repeated. Patients with suspected occult scaphoid fracture received bone scintigraphy on average 4 days $(1$ - 9) after the trauma. A scaphoid fracture was suspected when the clinical presentation was the following:

1) An observable swelling of the anatomical snuffbox.

2) Pain when applying pressure on the anatomic snuffbox or the scaphoid tubercle, or when applying axial pressure to the thumb or index fingers.

The results of bone scintigraphy of these patients are shown in Table 1. Outcome of bone scintigraphy: 80 $(18.0 \%)$ a scaphoid fracture, $145(32.6 \%)$ another fracture in the carpal region, 208 (46.7\%) normal and the diagnosis of 12 (2.7\%) was unclear. Figures 1 to 3 are images of three random selected patients. The diagnosis on bone scintigraphy was in some cases unclear, because clinical nuclear physicians described only deviation and didn't make statements about the diagnosis. In two cases they were not certain about the diagnosis.

Tabel 1. Outcome of bone scintigraphy in $\mathbf{4 4 5}$ patients with a suspected occult scaphoid fracture.

\begin{tabular}{ccc}
\hline Diagnosis & Number & Percent (\%) \\
\hline Scaphoid fracture & 80 & 18.0 \\
Lunate fracture & 2 & 0.45 \\
Triquetral fracture & 19 & 4.3 \\
Pisiform fracture & 2 & 0.45 \\
Trapezium fracture & 18 & 4.0 \\
Trapezoid fracture & 7 & 1.6 \\
Capitate fracture & 10 & 2.2 \\
Hamata fracture & 5 & 1.1 \\
Diastal radius fracture & 52 & 11.7 \\
Distal ulna fracture & 3 & 0.7 \\
Distal radius fracture and fracture of & 10 & 2.2 \\
a carpal bone & 1 & 0.2 \\
Distal ulna and distal radius fracture & 8 & 1.8 \\
Fracture of more carpal bones & 37 & 8.3 \\
Other (Metacarpal fracture and wrist & 179 & 40.2 \\
contusion) & 445 & 2.65 \\
Normal & & 100 \\
Unclear & Total & 179 \\
\hline
\end{tabular}
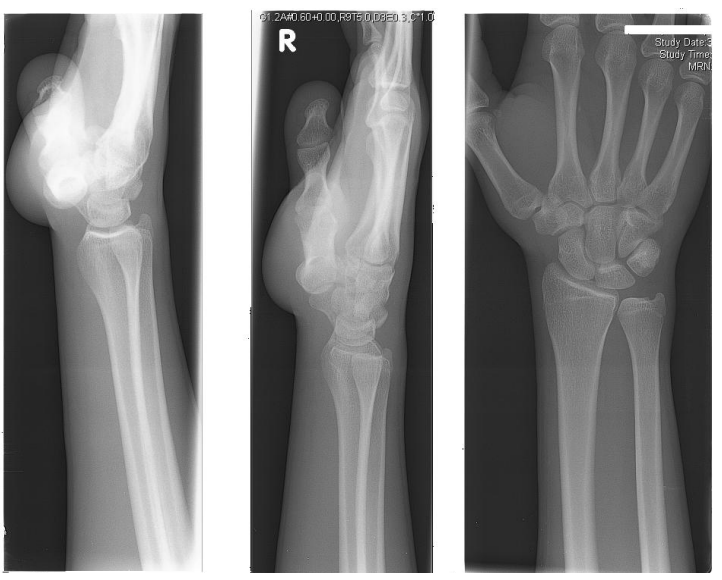

(a)

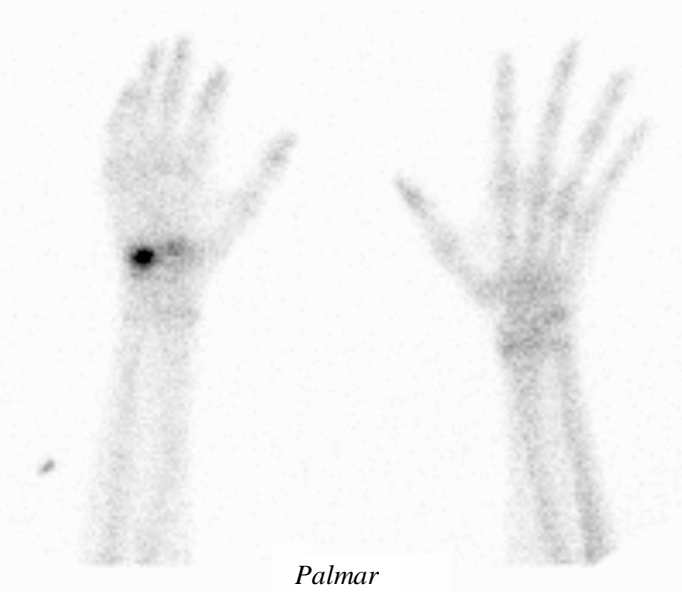

(b)

Figure 1. An example of a 19 years old man with suspected scaphoid fracture on the right side. (a) Wrist X-rays showing no fracture. (b) Bone scintigraphy showing hamata fracture.

\subsection{Imaging}

\subsubsection{Wrist Radiographs}

All radiographs were obtained by using a digital technique. Initial radiographs were taken in following 3 planes: 1) a posteroanterior view with the hand in a neutral position, 2) an oblique view with the wrist in 10 degrees of supination and maximal ulnar deviation, and 3) a true lateral view with the wrist resting in the ulnar position on the X-ray plate.

\subsubsection{Bone Scintigraphy}

Bone scintigraphy was performed by using a standard protocol of images of the early static phase, on a SKYLight gamma camera (Philips, Eindhoven, The Netherlands). Palmar and dorsal images of both wrists were performed between 2 and a half and 4 hours after the intravenous injection of $500 \mathrm{MBq}$ of Tc-99m-HDP (Tc-99m hydroxymethylene diphosphonate) visualizing 

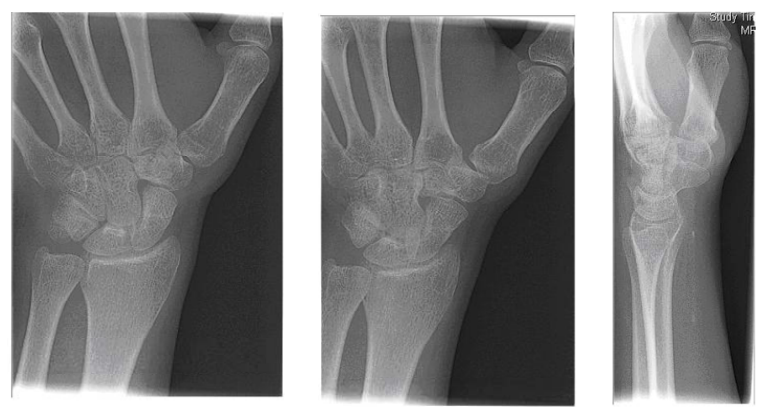

(a)

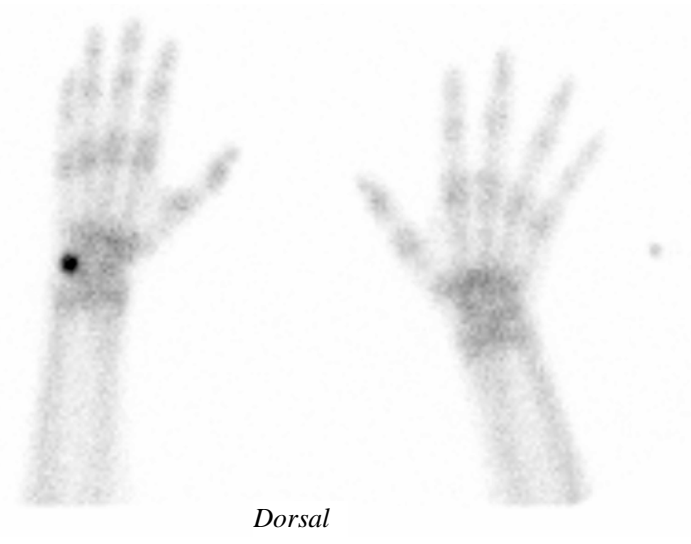

(b)

Figure 2. An example of a 20 years old woman with suspected scaphoid fracture on the left side. (a) Wrist X-rays showing no fracture. (b) Bone scintigraphy showing triquetral fracture.

the osteoblastic activity with a planar collimator. Each image took 10 minutes. A consultant clinical nuclear physician evaluated all bone scans. The reports of bone scans of the clinical nuclear physician are evaluated in this study.

\section{Discussion}

To this date, the main focus of occult fractures around the wrist was the scaphoid. However, analysis of this study showed that we should broaden our view. Our routine protocol of bone scintigraphy suspected scaphoid fracture revealed that over $60 \%$ fracture around the carpal region was identified, although only a scaphoid fracture was clinically suspected. It is generally accepted that advanced imaging modalities are needed in case of suspected scaphoid fractures and negative radiographs in order to rule out a scaphoid fracture. It is known that missed diagnosis and inadequate treatment of this fracture leads to nonunion and may cause pain and serious disability [7,10-14]. However, little is known on the clinical consequence of missing those other fractures which are detected in this study.

Those other fractures in the carpal region were not dislocated, so they would be treated conservatively. It is
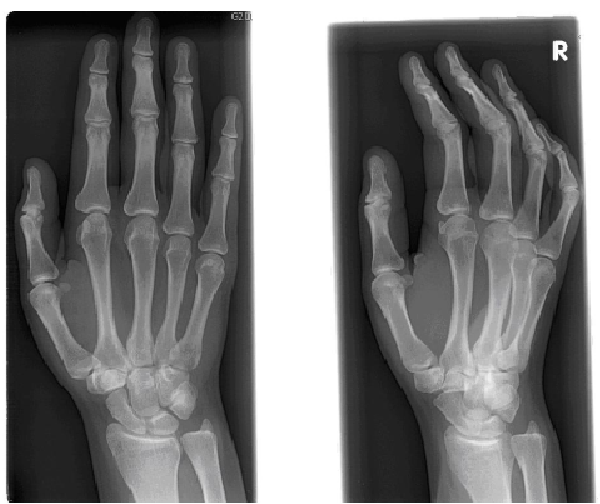

(a)

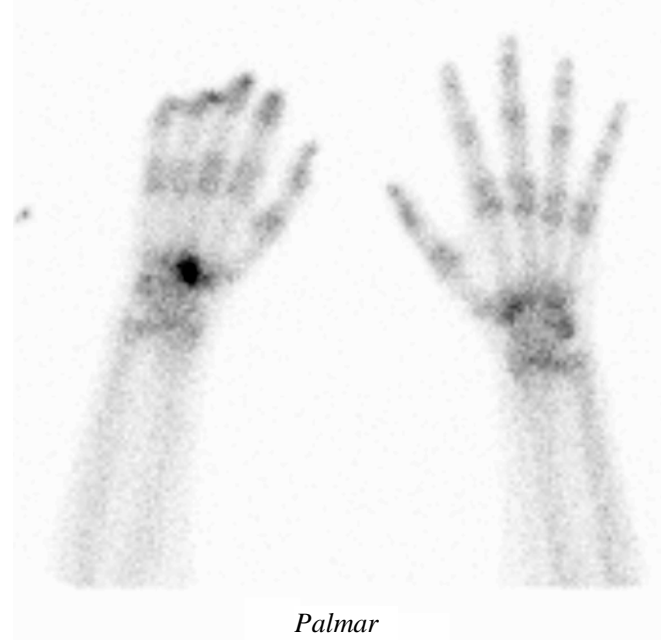

(b)

Figure 3. An example of a 19 years old woman with suspected scaphoid fracture on the right side. (a) Wrist $X$-rays showing no fracture. (b) Bone scintigraphy showing capitate fracture.

not quite clear what the consequence is for these patients with inadequate treatment, but it is likely that they will bring disability and that they will have socio-economic consequences. If the fracture had been detected by those patients, they would probably have had plaster for four to six weeks. In our society most people with plaster take rest and insurance pays when they are physically not able to work. Therefore we can assume that those patients with inadequate treatment could also not work for four to six weeks or even longer because of what could classify as a disability, pain and slowly recovery. But in this case the insurance is not paying because the fracture is not confirmed medically.

The study of Welling concluded that radiography failed to detect $30 \%$ of wrist fractures identified on CT, which suggests the importance of further advanced or cross-sectional imaging for fracture detection in the correct clinical scenario. In the proximal carpal row, lunate and triquetral fractures were often radiographically oc- 
cult ( $0 \%$ and $20 \%$, respectively, detected at radiography); whereas in the distal carpal row, trapezoid, capitate, and hamate fractures were often occult $(0 \%, 0 \%$, and $40 \%$ detected at radiography, respectively) [15]. The study of Pierre-Jerome identified with MR examination a high prevalence of multiple occult bone injuries in patients with clinically suspected scaphoid fractures but negative radiographs and persistent wrist pain [16]. Moreover, repeated radiographs do not lead to improvement of the diagnostic management, since the added sensitivity of repeated radiographs is low $[5,6,17]$. This also implies that repeated radiographs are not cost effective compared to other diagnostic modalities [18]. That is why we should focus on advanced or cross-sectional imaging to solve this problem.

The study of Querellou highlights the advantage of bone scintigraphy, which allows the detection of most occult carpal fractures and reduces the risks of complications such as pseudoarthritis [19]. Beeres suggests that bone scintigraphy is required when scaphoid X-rays do not confirm a suspected scaphoid fracture, even in the presence of other fractures in the carpal region [20]. The study of Akdemir concluded that bone scintigraphy is a reliable method to confirm or exclude the presence of a suspected scaphoid fracture and also useful to detect clinically unsuspected fractures of distal radius and other carpal bones [21].

We acknowledge that it is not the most wise decision to use nuclear imaging in the initial investigation of patients with wrist injury, because it is invasive, carries a varying specificity, it is time-consuming, costly and not routinely available. Additionally, further advanced or cross-sectional imaging (MRI and CT) are also timeconsuming, not routinely available, costly and they have been shown false negatives. Computed Tomography (CT) and bone scintigraphy have also another weakness, it have a radiation burden [22].

Bone scintigraphy has a sensitivity of up to $100 \%$ and a specificity of approximately $85 \%$ [7,20,23-26]. In addition, according to several reviews, a negative bone scan virtually excludes a scaphoid fracture. However, although sensitive, bone scan is not specific for a scaphoid fracture and may be positive due to other injuries, It may result in unnecessary immobilization due to fals positive results. Bone scintigraphy and CT are inferior to MRI for the detection of bone bruises and ligamentous injuries. Most occult fractures represent damage to the trabecular bone. The merit of MRI over bone scintigraphy is its high specificity in showing these lesions [16]. CT has a sensitivity of $64 \%$ and specificity of $99 \%$ [23]. MRI has a sensitivity of $80 \%$ and specificity of $100 \%[24,26]$. The weakness of CT and MRI is that they may show fals negatives results and so mis a fracture [27]. However these three modalities miss far fewer fractures than con- ventional radiography.

The low prevalence of true fractures amongst suspected scaphoid fractures magnifies the shortcomings of the diagnostic tests used to triage suspected scaphoid fractures. Therefore, clinical predictions rules are being investigated to yield a subset of patients who are more likely to have a fracture than others who laced the criteria. An initial study [28] regarding scaphoid fractures has been performed on this issue. However, we should broaden our view and see if we can make a clinical prediction rule for the hand and wrist.

There were several advantages to this study. The number of examined human subjects was high, all bone scans were evaluated by clinical nuclear physician and we made clear statements about the diagnosis on bone scintigraphies. Observer variation for bone scintigraphy could be suggested as a weakness, but a previous study at our institution showed that the observer variation is very small [29].

There are also some weaknesses as the study population had been derived from previous studies on scaphoid fractures. In addition, it cannot be verified how experienced the emergency room physicians were who evaluated the patients. However physical examination findings are not specific and may be seen in patients without any fractures or with fractures of the radial styloid, trapezium, carpal bones and metacarpal bones. It would have been interesting to evaluate the follow-up of these patients; however, this would be beyond the scope of this article. A bone scan with a interval of 4 days after injury will be "hot" due to a variety of reasons other than fracture- this includes inter-carpal ligament sprains and tears (inflamemation) after injury.

In the present study, we demonstrated that in patients with a clinically suspected scaphoid fracture and negative initial radiographs, bone scintigraphy detected in many cases $(64.4 \%)$ other fractures in the carpal region. This suggests that radiographs not only miss scaphoid fracture but also many other carpal and distal radius fractures. Solutions should be found to solve this problem and probably advanced imaging techniques like MRI, bone scintigraphy or CT should be used in the correct clinical scenario while keeping the financial impact in mind.

\section{Take Home Messages}

- Radiographs miss not only scaphoid fractures but also many other carpal and distal radius fractures.

- If you suspect an occult scaphoid fracture, you may find with a advanced imaging technique a fracture of a another bone in the carpal region.

- An advanced imaging technique like CT, MRI or bone scintigraphy is required for the clarification of the diagnosis and adequate treatment when there is a 
clinical suspicion of an occult fracture in the carpal region.

\section{REFERENCES}

[1] M. P. Eiff, R. L. Hatch and W. L. Calmbach, "Carpal Fractures,” In: Fracture Management for Primary Care, 2nd Edition, Saunders, Philadelphia, 2003, Chapter 5.

[2] J. L. Hobby, A. K. Dixon, P. W. Bearcroft, et al., "MR Imaging of the Wrist: Effect on Clinical Diagnosis and Patient Care,” Radiology, Vol. 220, No. 3, 2001, pp. 589593. doi:10.1148/radiol.2203001429

[3] W. C. G. Peh, L. A. Gilula and A. J. Wilson, "Detection of Occult Wrist Fractures By Magnetic Resonance Imaging,” Clinical Radiology, Vol. 51, No. 4, 1996, pp. 285292. doi:10.1016/S0009-9260(96)80348-5

[4] W. Bessler, "Scintigraphic Studies Following Fractures and Bone Surgery,” Radiologia Clinica et Biologica, Vol. 36, No. 5, 1967, pp. 275-276.

[5] M. M. Tiel-van Buul, E. J. van Beek, J. J. Borm, et al., "The Value of Radiographs and Bone Scintigraphy in Suspected Scaphoid Fracture. A Statistical Analysis,” Journal of Hand Surgery, Vol. 18, No. 3, 1993, pp. 403406. doi:10.1016/0266-7681(93)90074-P

[6] M. M. Tiel-van Buul, W. Roolker, A. H. Broekhuizen, et al., "The Diagnostic Management of Suspected Scaphoid Fracture,” Injury, Vol. 28, No. 1, 1997, pp. 1-8. doi:10.1016/S0020-1383(96)00127-1

[7] L. R. Bayer, A. Widding and H. Diemer, "Fifteen Minutes Bone Scintigraphy in Patients with Clinically Suspected Scaphoid Fracture and Normal X-Rays,” Injury, Vol. 31, No. 4, 2000, pp. 243-248. doi:10.1016/S0020-1383(99)00280-6

[8] M. M. Tiel-van Buul, E. J. van Beek, A. van Dongen, et al., "The Reliability of the 3-Phase Bone Scan in Suspected Scaphoid Fracture: An Inter- and Intraobserver Variability Analysis," European Journal of Nuclear Medicine, Vol. 19, No. 10, 1992, pp. 848-852. doi:10.1007/BF00168159

[9] W. P. Cooney, "Scaphoid Fractures: Current Treatments and Techniques,” Instructional Course Lectures, Vol. 52, No., 2003, pp. 197-208.

[10] E. Krasin, M. Goldwirth, A. Gold, et al., "Review of the Current Methods in the dIagnosis and Treatment of Scaphoid Fractures,” Postgraduate Medical Journal, Vol. 77, 2001, pp. 235-237. doi:10.1136/pmj.77.906.235

[11] G. H. Prosser and E. S. Isbister, "The Presentation of Scaphoid Non-Union,” Injury, Vol. 34, No. 1, 2003, pp. 65-67. doi:10.1016/S0020-1383(02)00162-6

[12] W. Roolker, M. Maas and A. H. Broekhuizen, "Diagnosis and Treatment of Scaphoid Fractures, Can Non-Union Be Prevented?" Archives of Orthopaedic and Trauma Surgery, Vol. 119, No. 7-8, 1999, pp. 428-431. doi:10.1007/s004020050014

[13] M. M. Tiel-van Buul, W. Roolker, A. H. Broekhuizen, et al., "The Diagnostic Management of Suspected Scaphoid Fracture,” Injury, Vol. 28, No. 1, 1997, pp. 1-8. doi:10.1016/S0020-1383(96)00127-1
[14] J. J. Nikken, Oei Eh, A. Z. Ginai, et al., "Acute Wrist Trauma: Value of a Short Dedicated Extremity MR Imaging Exination in Prediction of Need of Treatment," Radiology, Vol. 234, No. 1, 2005, pp. 116-124. doi:10.1148/radiol.2341031067

[15] R. D. Welling, J. A. Jacobson, D. A. Jamadar, et al., "MDCT and Radiography of Wrist Fractures: Radiographic Sensitivity and Fracture Patterns," American Journal of Roentgenology, Vol. 190, No. 1, 2008, pp. 10 16. doi:10.2214/AJR.07.2699

[16] C. Pierre-Jerome, V. Moncayo, U. Albastaki, et al., "Multiple Occult Wrist Bone Injuries and Joint Effusions: Prevalence and Distribution on MRI,” Emergency Radiology, Vol. 17, No. 3, 2010, pp. 179-184. doi:10.1007/s10140-009-0827-5

[17] C. Gaebler, C. Kukla, M. Breitenseher, et al., "Magnetic Reso-Nance Imaging of Occult Scaphoid Fractures," Journal of Trauma, Vol. 41, No. 1, 1996, pp. 73-76. doi:10.1097/00005373-199607000-00011

[18] M. M. Tiel-van Buul, E. J. van Beek, J. J. Borm, et al., "The Value of Radiographs and Bone Scintigraphy in Suspected Scaphoid Fracture. A Statistical Analysis," Journal of Hand Surgery, Vol. 18, No. 3, 1993, pp. 403406. doi:10.1016/0266-7681(93)90074-P

[19] S. Querellou, G. Moineau, A. Le Duc-Pennec, et al., "Detection of Occult Wrist Fractures by Quantitative Radioscintigraphy: A Prespective Study on Selected Patients," Nuclear Medicine Communications, Vol. 30, No. 11, 2009, pp. 826-827. doi:10.1097/MNM.0b013e3283306f87

[20] F. J. Beeres, M. Hogervorst, P. Den Hollander, et al., "Diagnostic Strategy for Suspected Scaphoid Fractures in the Presence of Other Fractures in the Carpal Region," Journal of Hand Surgery, Vol. 31, No. 4, 2006, pp. 416418. doi:10.1016/j.jhsb.2006.04.007

[21] U. O. Akdemir, T. Atasever and S. Siphioglu, "Value of Bone Scintigraphy in Patients with Carpal Trauma," Annals of Nuclear Medicine, Vol. 18, No. 6, 2004, pp. 495499. doi:10.1007/BF02984566

[22] E. J. Hall and A. J. Giaccia, "Radiobiology for the Radiologist," 6th Edition, Lippincott Williams \& Wilkins, Philadelphia, 2006, Chapter 14.

[23] J. S. Rhemrev, Andele D. de Zwart and L. M. Kingma, "Early Computed Tomography Compared with Bone Scintigraphy in Suspected Scaphoid Fractures," Clinical Nucleard Medicine, Vol. 35, No. 12, 2010, pp. 931-934. doi:10.1097/RLU.0b013e3181f9de26

[24] F. J. Beeres, S. J. Rhemrev and P. den Hollander, "Early Magnetic Resonance Imaging Compared with Bone Scintigraphy in Suspected Scaphoid Fractures,” The Bone \& Joint Journal, Vol. 90, No. 9, 2008, pp. 1205-1209. doi:10.1302/0301-620X.90B9.20341

[25] C. Gaebler, C. Kukla, M. Breitenseher, et al., "Magnetic Resonance Imaging of Occult Scaphoid Fractures,” The Journal of Trauma, Vol. 41, No., 1996, pp. 73-76. doi:10.1097/00005373-199607000-00011

[26] C. Fowler, B. Sullivan, L. A. Williams, et al., "A Comparison of Bone Scintigraphy and MRI in the Early Di- 
agnosis of the Occult Scaphoid Waist Fracture,” Skeletal Radiology, Vol. 27, No. 12, 1998, pp. 683-687.

doi:10.1007/s002560050459

[27] A. M. Groves, H. K. Cheow, K. K. Balan, et al., "Falsenegative 16-Detector Multislice CT for Scaphoid Fracture,” British Journal of Radiology, Vol. 78, 2005, pp. 5759. doi:10.1259/bjr/55015850
[28] S. J. Rhemrev, F. J. P. Beeres, R. H. van Leerdam, et al., "Clinical Prediction Rule for Suspected Scaphoid Fractures,” Injury, Vol. 41, 2010, pp. 1206-1030.

[29] J. P. Beeres, M. Hogervorst, S. J. Rhemrev, et al., "Reliability of Bone Scintigraphy for Suspected Scaphoid Fractures," Clinical Nuclear Medicine, Vol. 32, No. 11, 2007, pp. 835-838. doi:10.1097/RLU.0b013e318156bb91 